\title{
Les origines de l'imaginaire bauchalien. Quelques aperçus génétiques de Géologie
}

\begin{abstract}
C'est que le temps ne passe pas vraiment. Il persiste, en nous, à proportion de ce qu'on n'a pu lui être présent dans toute la mesure où cela se pouvait, où on le voulait, quand c'était le moment. Des choses nous ont nui pour garder leur secret. Elles ne nous ont pas dit quelles elles étaient. Et alors on n'a pu être soi-même. Une part de ce qui nous affecte et en quoi, par suite, on consiste, est restée entre leurs mains et nous a donc manqué, diminués. ${ }^{1}$
\end{abstract}

\section{Présentation du dossier de genèse}

S'intéresser aux archives du recueil Géologie, c'est en quelque sorte partir en quête d'une quête. Partir en quête, tout d'abord, car loin d'être un ensemble homogène, géographiquement et topographiquement identifiable, les brouillons et divers éléments constitutifs du dossier de genèse sont au contraire éparpillés et nécessitent d'être débusqués par le chercheur persévérant. Mais cette quête est avant tout la mise en lumière d'une autre quête : celle d'Henry Bauchau lui-même qui, délaissant au sortir de la guerre son action politique, et suivant ainsi la voix de la Sibylle, s'engage dans une vocation de poète dont il va, dans cette première œuvre, chercher les racines au plus profond de lui. Dégager et souligner, par le biais du travail poétique en action, c'est-à-dire par une attention minutieuse portée à un cheminement scriptural qui s'édifie pas à pas, les processus de mise en œuvre et d'accomplissement de cette quête, tel sera l'objet de la présente recherche.

La constitution du dossier de genèse de Géologie est conséquente: celui-ci se compose de plus de cinq cents folios d'avant-textes qui

\footnotetext{
${ }^{1}$ Pierre Bergounioux, Géologies, Paris, Galilée, «Incises », 2013, pp. 43-44.
} 
correspondent aux brouillons des poèmes publiés dans l'édition Gallimard de $1958^{2}$, mais aussi aux divers brouillons et mises au net de poèmes écrits entre 1949 et 1957 (période de rédaction du matériau poétique du volume, initiée par le poème "Les sandales éclatantes »), mais finalement non retenus pour la publication (certains de ces poèmes feront cependant leur apparition dans des recueils plus tardifs, ou lors de rééditions $\left.{ }^{3}\right)$. Ces diverses archives sont réparties sur deux sites. D’une part, le Fonds Henry Bauchau des Archives et Musée de la Littérature (Bruxelles) dispose d'un cahier noir relié en tranche bleu foncé, non daté (mais dont la confection peut être estimée à 1956 ou 1957') et intitulé Les Chevaux de la mer. Il s'agit d'un tapuscrit comportant quelques corrections manuscrites qui constitue un premier état du recueil à venir (ML07160/0005). De plus, les A.M.L. possèdent également trois folios (ML09451/0001-0003), brouillons manuscrits du «Cantique de l'Attente », et, surtout, une liasse de plus de deux cents folios manuscrits et tapuscrits, qui correspondent à des brouillons des poèmes de ce qui est alors dénommé Géologies (ML07160/0007).

D'autre part, le Fonds Henry Bauchau de l'Université catholique de Louvain détient de nombreux brouillons des poèmes édités dans le recueil de 1958, mais aussi des textes rédigés concomitamment et non publiés (il s'agit des folios A1 à A21, d'environ deux cents folios disséminés à la fin du dossier 6 [A2025bis-A2081], dans le dossier 7 [A2081bis-A2382bis] et au début du dossier 39 [A14865-A15065], et d'un cahier de travail manuscrit noir [A21-342-A21423]). Par ailleurs, le Fonds Henry Bauchau possède également une «frappe préliminaire » du recueil (cahier tapuscrit noir relié en tranche bleu foncé A21466-A21556) ainsi qu'un ensemble de feuillets tapuscrits (A21302-A21340), poèmes écrits à l'époque de Géologie, non retenus pour le manuscrit et révisés par l'écrivain en 2005 dans l'optique d'une éventuelle publication.

Outre ces avant-textes, le dossier de genèse comporte plusieurs articles de presse consacrés à la publication du recueil (notamment lors

\footnotetext{
${ }^{2}$ Henry Bauchau, Géologie, Paris, Gallimard, « Métamorphoses », 1958.

3 C'est, par exemple, le cas des poèmes "Sèvres-Babylone » (ca. 1950) et "Chanson dauphine » (1951), tous deux publiés en 2006 dans le recueil Nous ne sommes pas séparés.

${ }^{4}$ Certains poèmes présents dans le tapuscrit sont en effet datés de 1956.
} 
de son attribution du Prix Max Jacob) ${ }^{5}$, une série de huit cahiers d'écolier (ML08872/0001-0008) numérotés et datés (entre juin 1954 et juin 1959), journaux (inédits) accompagnant la création de Géologie (et de Gengis Khan) qui permettent de mieux cerner l'esprit anxieux dans lequel s'est opérée cette première écriture littéraire ${ }^{6}$. Enfin une correspondance vient enrichir le dossier de genèse, laissant la place à des destinataires aussi variés que Clément Lialine o.s.b. ${ }^{7}$ (du monastère bénédictin de Chevetogne $^{8}$ ), Gaston Bachelard" (qui cite un vers issu de "L'arbre de Gengis Khan » dans sa Poétique de la rêverie, publiée en $1960^{10}$ ), ou encore Philippe Jaccottet ${ }^{11}$ (dont on connait le rôle de soutien déterminant pour les débuts littéraires d'Henry Bauchau).

\section{Le choix d'un titre}

La première considération que l'on peut formuler à la lumière du dossier de genèse concerne le titre du recueil. Les avant-textes attestent d'une hésitation entre quatre titres. Le premier, dont témoigne le cahier déposé aux A.M.L., est Les Chevaux de la mer. Il s'agit en fait du premier hémistiche du premier vers du poème "Mélopée viking », dont la suite est «n'auront pas de poulains ». D'emblée ce choix met en évidence l'une des particularités majeures du recueil, à savoir l'imprégnation mythologique nordique et ce qu'elle draine en termes d'imaginaire

\footnotetext{
5 Voir, par exemple, Philippe Jaccottet, «Henry Bauchau: Géologie», dans La Nouvelle Revue française, août 1958, pp. 324-326 ; Adrien Jans, " Henry Bauchau, prix Max Jacob », dans Revue générale belge, 95/5, mai 1959, pp. 117-118.

${ }^{6}$ Il est à noter que le Fonds Henry Bauchau de Louvain-la-Neuve possède lui aussi quelques folios de ce journal, numérotés de A4252 à A4257 et de A4517 à A4525.

7 Il s'agit de deux lettres non foliotées, datées des 4 et 24 juin 1956, appartenant au Fonds Henry Bauchau.

8 Né en 1901 à Saint-Pétersbourg et décédé en 1958, Constantin Nicolaevitch Lialine était un intellectuel russe, converti au catholicisme, à Maredsous, en 1929. Grand connaisseur de la religion orthodoxe et du patrimoine de l'Église d'Orient, il fut longtemps collaborateur de la revue Irénikon, revue théologique œcuménique trimestrielle des moines de Chevetogne. Voir Olivier Rousseau, «Lialine (Clément), bénédictin, 1901-1958 », dans Marcel Viller, Ferdinand Cavallera et Joseph de Guibert (dir.), Dictionnaire de la spiritualité ascétique et mystique: doctrine et histoire, t. 9 (LabadieLyonnet), Paris, Beauchesne, 1976, pp. 749-750.

${ }^{9}$ B505-B506.

${ }^{10}$ Gaston Bachelard, La Poétique de la rêverie, Paris, PUF, « Quadrige », 2005, p. 162.

11 B2944 à B2854.
} 
bauchalien dans le volume. Car le "peuple de la mer» est celui des envahisseurs, celui de la «Caste $»^{12}$ des bourreaux guerriers qui fait écho de manière explicite aux événements de la décennie précédente. Mais si ce vers, sur lequel nous reviendrons plus en détails, laisse entendre l'impasse politique de cette force noire, il apparaît que cette dernière se révèle, au contraire, féconde sur le plan poétique. Ainsi, la pulsion dionysiaque à l'œuvre est-elle à l'origine de l'engendrement d'une inspiration de type cathartique qui jaillit et se déploie, pour reprendre le titre d'un essai de Jacques Brault, « dans la nuit du poème $»^{13}$.

Aussi la perspective induite par ce premier titre souligne-t-elle les résonances du monde contemporain à l'intérieur du poème, qui s'attache pour lors à en dégager le sens par la recherche de ses Géologies (autre titre envisagé par Bauchau pour son recueil). Remonter jusqu'aux racines des pulsions qui se déchaînent au sein du monde moderne, c'est bien là un autre aspect de l'ouvrage ${ }^{14}$. Toutefois, la poésie bauchalienne est essentiellement une poésie de l'affectivité, si bien que, selon un principe d'analogie qu'apprécie le poète, ce qui se joue au-dehors est à l'image de ce qui se joue au-dedans. Un mouvement s'opère depuis les géologies du monde à la Géologie de soi, depuis la pluralité des profondeurs obscures jusqu'à l'obscure profondeur de la pluralité du soi ${ }^{15}$. C'est l'homme face à lui-même qui se dévoile dans le poème, l'individu au regard de son intériorité la plus intime. La poésie d'Henry Bauchau côtoie ainsi celle de Pierre Jean Jouve - une parenté de ton que souligne Philippe Jaccottet lorsqu'il écrit, dans la critique qu'il accorde à Géologie dans la Nouvelle Revue française, que le poète belge "s'enfonce en lui-même, sur les traces de Jouve, cherchant à dominer les secrètes noirceurs qui l'entravent $»^{16}$. Et sans doute cet autre extrait, issu de Sueur de sang (publié par Jouve en 1933) et que reprend Bauchau dans "L'écriture à l'oreille enfantine ", n'est-il pas anodin dans le choix définitif du titre de son premier recueil : «Nous avons connaissance à présent de milliers de mondes à l'intérieur du monde de l'homme, que toute l'œuvre de l'homme avait été de

\footnotetext{
12 Voir le poème « Caste des guerriers » (G, pp. 17-18).

${ }^{13}$ Jacques Brault, Dans la nuit du poème, Montréal, Noroit, " Chemins de traverse », 2011.

${ }^{14}$ Voir notre article "Le récit mythique dans Géologie : l'émergence d'une parole-trace ", dans Revue internationale Henry Bauchau, n4, 2012, pp. 149-159.

${ }^{15}$ Sur la pluralité du Moi et l'unité du Soi, voir Carl Gustav Jung, L'Âme et la vie, Paris, Librairie générale française, «Le Livre de Poche. Références », 2012.

${ }^{16}$ Philippe Jaccottet, «Henry Bauchau : Géologie », op. cit., p. 325.
} 
cacher, et de milliers de couches dans la géologie de cet être terrible » (EE, p. 126). L'homme, cet «être terrible», sonde profondément en lui et s'achemine en quête de ce substrat originel au cœur duquel l'enfance jaillit, charriant avec elle l'inévitable mélancolie dont le regard en arrière la pare.

Le thème de l'enfant envahit littéralement le recueil. Plus qu'un simple motif, l'enfant est mythifié et est constamment placé sous le signe de la pureté, de la candeur, de la proie potentielle à protéger. Dans le poème «Enfants » apparait «l'enfant David qui jouait de la lyre » $(G$, p. 12), image prototypique (David est un jeune berger) appelé (il vainc Goliath et fait fuir les Philistins) et injustement réprouvé (il souffre la vindicte du roi Saül). Dans «La prière d'Ibrahim», il est question de "[l]'enfant sombre qui saigne » et qui «est l'élu» ( $G$, p. 13). «Negro spiritual» évoque «[t]ous les coquelicots des enfants innocents» $(G, \mathrm{p} .51)$. Les brouillons révèlent que le poème "Chemin des sources» s'est d'abord intitulé «Enfantine» (A14876-A14878), et celui-ci parle de «l'habile à manier les lents rideaux d'enfance [...] par les grands corridors aux chambres du passé» (G, pp. 39-40). Enfin, le texte "Les feux verts», primitivement titré "L'enfant liberté », fait allusion à "l'ombre où nous attend notre mort», mais où se distingue aussi «un visage enfant et brûlant qui [nous] guide » (G, p. 55).

En sa pleine innocence, l'enfance se présente dans Géologie dans la mélancolie d'un paradis perdu : «Ma honte [écrit Bauchau] fut d'errer si longtemps [...] quand l'ombre m'attirait par l'enfantin scandale » (A2058). L'enfance est un pur état prélapsaire inatteignable, elle est un «scandale », ce sur quoi vient achopper le poète qui, partant à sa quête, comprend son erreur: «Ma profondeur est la mémoire d'eaux plénières où j'ai voulu dormir toujours et refuser la violente inspiration » (idem; nous soulignons). C'est donc pour contester ce refus de l'inspiration que l'écrivain se met en chemin. Plus encore sa destination est celle d'une renaissance: "J'entends toujours mes pas d'enfant / Les pas tremblants dans les greniers du mal de naittre » $(G$, p. 35$)$. L'anamnèse à laquelle se livre le poète revient à effacer les barrières du temps et ses rêveries sur l'enfance l'aident «à descendre si profondément en [lui] qu'elles [le] débarrassent de [son] histoire. Elles [le] libèrent ${ }^{17}$. Car les liens par

${ }^{17}$ Gaston Bachelard, La Poétique de la rêverie, op. cit., p. 84. 
lesquels le poète se sent contraint sont précisément ceux de son histoire. $\mathrm{Ou}$, plus exactement, ce sont les dissonances entre l'individu présent et l'individu dans son temps passé qui créent cet état de discordance intime. Jung, que Bauchau a beaucoup lu, insiste dans L'Essence de la mythologie sur le fait que l'émergence de la figure mythifiée de l'enfant est souvent suscitée par des «incompatibilités, quand par exemple l'état actuel se trouve être en contradiction avec celui de l'enfance. On a pu, peut-être, se séparer violemment de son caractère originel au profit d'une individualité autre, arbitrairement choisie pour répondre à ses ambitions. En agissant ainsi, on est devenu artificiel, privé de sa nature enfantine, et de ce fait on a perdu ses propres racines. C'est l'occasion la plus propice pour une confrontation violente avec la vérité initiale $»^{18}$.

Or, cette " confrontation violente », dont témoigne Chemin sous la neige, est bien celle dans laquelle se trouve Henry Bauchau au sortir de la guerre, devant faire son deuil du statut «artificiel» de l'homme d'action qu'il n'est pas afin de rejoindre "son caractère originel», "sa nature enfantine » vers laquelle le guide la voix de la Sibylle : celle de créateur.

Tout le travail du poète consiste dès lors à donner voix à cet esprit d'enfance afin de lui permettre d'émerger des cavernes où il avait été enfoui. C'est cette mise en voix, c'est-à-dire aussi cet apprivoisement des pulsions les plus lointaines par l'entremise de l'écriture, sur lequel le titre Chansons pour Merveillee met l'accent. Le rythme du poème porte en lui la possibilité d'une révélation. C'est ce qu'exprime le texte inédit «Imprimé sur le voile»(A2349-A2350), dans lequel l'acte poétique est compris comme un dévoilement progressif à soi-même : «Il n'y a rien / que cet enfant qui dort et ne se connaît pas ». Telle est en définitive la quête à laquelle se livre le poète : éveiller l'enfant endormi en lui, et sans lequel il n'est «rien», il "ne se connait pas", ainsi que le soulignent les deux marqueurs de négation présents en fin de vers. Alors survient la «merveille », qui frappe d'étonnement: la surprise de constater que l'on n'est plus qui on est vraiment - mais l'espérance aussi, pour Bauchau, de pouvoir renouer avec la "vocation », aux sens propre et étymologique, et devenir le poète « natif de [s]es ruines surgissantes » $(P C, \mathrm{p} .151)$.

\footnotetext{
18 Voir Carl Gustav Jung et Charles Kerényi, L'Essence de la mythologie. L'enfant divin, la jeune fille divine, Paris, Payot, «Petite Bibliothèque Payot», 1980, p. 119.

19 Titre donné aux brouillons de Géologie déposés au Fonds Henry Bauchau.
} 


\section{Une structure malmenée}

Si l'on considère généralement l'année 1958, date de la publication de Géologie, comme l'entrée d'Henry Bauchau dans le champ de la littérature française contemporaine, il faut cependant noter la publication préalable de quelques textes, qui introduit d'ores et déjà le poète - avec la revendication expresse de ce statut - dans le milieu littéraire. En 1954, le Pays du Lac, cahier de poésie suisse dont le responsable est Jacques Chessex, accueille les poèmes «Les Pleureuses » et «Les Mongols bleus $»^{20}$ (Bauchau est alors en pleine écriture de Gengis Khan). Ces deux textes seront repris et accompagnés de "Corne des lassitudes»: tous trois formeront les Trois chansons d'Asie amère (finalement intégrées dans la section Caste des guerriers) que l'écrivain verra publiées en 1956 dans La Nouvelle Revue française ${ }^{21}$. Cette première consécration place l'écrivain aux côtés de personnalités comme Maurice Blanchot, Jean Grosjean, André Pieyre de Mandiargues ou encore Christian Dotremont. Elle sera suivie, deux ans plus tard, par l'édition d'un recueil dans la collection «Métamorphoses », dirigée par Jean Paulhan chez Gallimard ${ }^{22}$, volume qui sera distingué par le Prix Max Jacob en $1959^{23}$. Le recueil Géologie sera ensuite repris, avec des variantes, dans les trois rééditions des œuvres poétiques: Poésie (1986), Heureux les Déliants (1995) et Poésie complète (2009).

Quelles leçons peut-on tirer d'une analyse comparée de ces différentes (ré)éditions? Une première démarche, qui consiste en l'analyse macrostructurale $\mathrm{du}$ recueil (organisation des sections et répartition des poèmes à l'intérieur de ces dernières), permet de dégager l'une des grandes caractéristiques de la «méthode» poétique

\footnotetext{
20 «Les pleureuses » et «Les Mongols bleus », dans Pays du Lac. Cahiers de poésie, série 2, no1, 1954, pp. 47-48.

21 Trois chansons d'Asie amère ("Les pleureuses », "Les Mongols bleus », "Corne des lassitudes »), La Nouvelle Revue française, n46, 1956, pp. 748-749.

${ }^{22}$ Collection dans laquelle on retrouve, entre autres, Le Parti pris des choses de Francis Ponge, Oublieuse mémoire de Jules Supervielle, Prospectus aux amateurs de tout genre de Jean Dubuffet ou encore Le Théâtre et son double d'Antonin Artaud.

${ }^{23}$ Le recueil fera en outre l'objet d'une reproduction anastatique par les éditions Gallimard en 2009.
} 
bauchalienne: chacune des rééditions successives de recueils poétiques de l'écrivain entraîne la relecture et la remise des textes sur le métier ${ }^{24}$.

Tapuscrit 1956-1957

Caste des guerriers

Trois chansons d'Asie amère

Tiers état

Chemins

Chants pour entrer dans la ville

Tombeaux pour des archers

Réédition Actes Sud 1986

Géologie

Caste des guerriers

Trois chansens d'Asie

amère

Tiers

Ehemins

Tombeaux pour des archers

Chants pour entrer dans la ville

Double Zodiaque

$<$ Deux chansons $>$
Édition Gallimard 1958

Caste des guerriers

Trois chansons d'Asie amère

Tiers état

Chemins

Chants pour entrer dans la ville

Tombeaux pour des archers

$<$ Double Zodiaque $>$

$<$ Géologie $>$

Réédition Actes Sud 2009

Géologie

Caste des guerriers

Tombeaux pour des archers

Chants pour entrer dans la ville

Double Zodiaque

Deux chansons

\section{Légende}

Suppression< <ajout> déplacement

Euvre de la jeunesse (littéraire) de l'écrivain, on peut supposer que Géologie fait montre pour Bauchau d'une maturité poétique qui se cherche, ce qui explique que ce soit le recueil qu'il a le plus remanié. Les ajouts ou suppressions de vers, de poèmes, voire de sections témoignent ainsi du constant souci qu'a le poète de revenir sur ses écrits et provoquent une forte perturbation de l'organisation interne, et donc un réaménagement de sa cohérence globale. Il faut d'abord noter l'importance capitale de "Géologie ». Poème tardif, son écriture va entraîner une réelle refonte du recueil, qui, jusqu'alors, s'organise

\footnotetext{
24 À l'exception de Heureux les Déliants. Poèmes 1950-1995 qui, à côté de textes poétiques neufs, reprend les textes proposés dans Poésie. 1950-1986, tout en inversant cependant l'ordre chronologique.
} 
essentiellement autour de la thématique guerrière, comme en témoigne le tapuscrit de 1956-1957, qui place les sections Caste des guerriers et Tombeaux pour des archers respectivement en début et fin de volume. Dès sa rédaction, "Géologie » devient le poème qui va donner son ton au recueil, si bien qu'il lui fournit son titre définitif. De plus, alors qu'il se situe en fin de recueil dans l'édition originale, Bauchau décide d'en faire le poème d'ouverture de Géologie et, de facto, de l'ensemble de son entreprise poétique, lors de la réédition de 1986.

Une autre constatation est la disparition de la section Chemins dans la réédition de 1986. Cette suppression est surprenante dans la mesure où le «cheminement» (personnel) représente l'un des axes majeurs qui sous-tendent Géologie, ainsi que le montrent les titres choisis, systématiquement formés à partir du syntagme "Chemin de + nom », et ainsi que le révèlent également le poème «Chemin du monde » (A2150), que le poète choisira finalement de ne pas intégrer au recueil, et les titres originellement pressentis pour le poème "Géologie», soit "Sans Chemin », "Chemin des arbres» et "Chemin de sève »" Or, cette thématique ne s'efface pas tout à fait: Bauchau n'ôte de la réédition de 1986 que le texte "Chemin de terre» et transfère en fait «Chemin d'enfance », "Chemin des sources » et "Chemin d'Héraclite» dans la section Chants pour entrer dans la ville. Aussi le cheminement est-il toujours présent (ce que confirmera de façon patente CEdipe sur la route en 1990), mais Bauchau préfère lui accorder une place en filigrane dans l'œuvre, laissant au lecteur le soin de la découverte de son importance.

\section{L'éludement du sens explicite}

Cette décision répond à un autre grand principe qui gouverne la réécriture bauchalienne : celui de l'éludement du sens explicite. Le poète tend dans ses brouillons vers un gommage des références trop nettes et préfère souvent un effacement des contours du sens, qui laisse à l'interprétation du lecteur davantage de latitude, tout en offrant à la matière poétique une portée plus intemporelle et plus universelle. Ainsi, dans le poème "Enfants », Bauchau décide lors de la réédition de 1986 de supprimer le vers "Adolescents noircis, garçons, garçons d'Hitler », qui renvoie expressément aux jeunesses hitlériennes, et maintient une

${ }^{25}$ Voir ML07160/0007. 
suspension du sens en ne gardant que le vers qui précède «Enfants martyrs, enfants bourreaux, enfants perdus ». De la même façon, il supprime de Géologie les éléments à portée clairement autobiographique ${ }^{26}$, alors même que ceux-ci sont passés par le prisme de son imaginaire. C'est pourquoi est laissé de côté, au moment de la publication, le poème "Chemin du monde » (également intitulé «Les genêts ») ${ }^{27}$, qui constitue une première ébauche de l'épisode de l'incendie de Sainpierre, et que Bauchau décide finalement de retirer lors de la réédition de 1986 le poème «Sibylle de la nuit» (G, pp. 45-46), qui évoque son expérience psychanalytique avec Blanche Reverchon-Jouve. Les rééditions successives voient également disparaitre progressivement le terme «Caste» (de la "Caste des guerriers»), dont la majuscule rappelle peut-être trop évidemment le terme allemand «Kaste »: tandis que, dans la réédition de 1986, Bauchau ôte du poème «Enfants » le vers «La Caste a revêtu la robe sans couture », il va jusqu'à décider de supprimer dans la réédition de 2009 le poème "Caste des guerriers », qui pourtant donne son titre et explicite, en quelque sorte, la section qui l'accueille et qui représentait, dans le tapuscrit de 1956-1957 le poème situé à l'initiale du volume.

Une autre suppression pouvant se comprendre dans la perspective d'un éludement du sens est celle de la section Tiers état, enlevée du recueil dès la réédition de 1986. Comme pour Chemins, si la section disparait, deux des trois poèmes qui la composent, "Pilate» (renommé "Selon Pilate ») et «Europe», sont replacés dans les Chants pour entrer dans la ville - seul le texte «Les enfants de fornication » est définitivement soustrait. Construite sur l'image des États généraux de l'Ancien Régime que le poète transpose dans le contexte des années 1930 et 1940, cette section propose une critique des trois grandes parties en présence que sont l'Europe, l'Église et les «enfants de fornication». Cependant que l'Europe percluse se distingue sous les traits d'une "génération [qui] ressemble à des enfants qui se tourmentent / En répétant avec ennui » $(G$, p. 27$)$ : «ainsi notre jeunesse est morte» $(G$, p. 28), l'Église s'incarne, sous la plume du poète, dans la figure de Ponce Pilate - une assimilation que renforce l'allitération en [p], à l'heure où le pape se nomme Pie XI ou Pie XII - qui, «se lavant

\footnotetext{
${ }^{26}$ Ceux-ci formeront le matériau de recueils ultérieurs, comme L'Escalier bleu (1964) ou La Sourde oreille ou le rêve de Frend (1981).

${ }^{27}$ Alors que le poème était initialement intégré au recueil, comme le montre le tapuscrit de 1956-1957. Voir FHB A2150, A2334 et A14908 à A14912.
} 
les mains ", annonce au dernier vers du poème : "que la vérité est triste » ( $G$, p. 30). Ce blâme de Bauchau à l'égard de la position du Vatican qu'il ne trouve pas suffisamment tranchée n'est pas neuf. Myriam WattheeDelmotte, dans Henry Bauchau dans la tourmente du XX siècle, le remarquait déjà dans un texte poétique daté de 1936, dans lequel le poète pointait l'Église qui «dort comme jadis au jardin des olives » ${ }^{28}$. Quant à «l'enfant de fornication », « [c] et ange noir à la folie », il laisse transparaitre la figure d'Hitler, que Bauchau qualifie par ailleurs dans La Sourde oreille ou le rêve de Freud de "Prométhée noir» (PC, p. 240). "Noir à la folie», il fait se déchaîner sur la vieille Europe ses «enfants» «qui s'aiment [dans la] mélancolie» d'une race en laquelle il leur fait croire par infidélité ${ }^{29}$. Le terme «fornication" possède en effet une seconde acception, relative à l'histoire du peuple juif, qui renvoie à l'«infidélité du peuple juif qui abandonne Dieu pour adorer des dieux étrangers ». Bauchau reprend ce sens figuré qu'il détourne en l'accolant au bourreau alors qu'il appartient à la tradition culturelle de la victime - un emploi qui a pour effet de renforcer la culpabilité du tortionnaire. En des pulsions assassines, tous s'entrainent en une «fornication" sanglante où «l'on entend sur les charniers / Voler les guêpes du silence » («Les Mongols bleus », G, p. 22). Il n'est donc pas étonnant que soient supprimés cette section et ce dernier texte peut-être trop explicites aux yeux du poète.

D'autres amputations sont encore à signaler. C'est le cas du «Cantique de l'attente », qui s'accorde tout à fait à la tonalité du recueil, mais qui fut rédigé dans un esprit différent. Daté de 1932, ce texte déborde le cadre temporel fixé par le poète lui-même pour l'élaboration de son matériau poétique et qui couvre les années 1949-1957. En effet, dans son journal de création, l'auteur note, à la date du 24 août 1961, pendant qu'il travaille à la rédaction de La Déchirure: «Derrière la muraille se trouvent les deux maisons. La chaude et la froide. Peut-être pour indiquer la percée faut-il placer là "Le chant du bélier" et la naissance du premier poème, "Les sandales éclatantes"» (GM, p. 151 ; nous soulignons). En effet, si «Les sandales éclatantes » est finalement resté inédit (il n’est

\footnotetext{
${ }^{28}$ Geneviève Duchenne, Vincent Dujardin et Myriam Watthee-Delmotte, Henry Bauchau dans la tourmente $d u X X^{e}$ siècle. Configurations historiques et imaginaires, Bruxelles, Le Cri, «Biographie », 2008, p. 137. Voir le tapuscrit en question, intitulé « C'est ton destin...» (FHB A2204).

${ }^{29}$ Trésor de la langue française en ligne, http:/ / atilf.atilf.fr (page consultée le 18 juin 2013).
} 
paru ni dans Géologie, ni dans La Déchirure), il porte néanmoins en germe les thématiques et les motifs dont s'apprêteront les poèmes ultérieurs : «les naseaux en feu [qui] lancent leur cri lunaire / Étalons! », rappelle le poème inédit «Palais Vert ${ }^{30}$; le «bruit du désir» est aussi celui de Géologie, où, écrit Bauchau à la date du 30 mai 1956, « la pulsion profonde reste presque exclusivement celle de l'Éros, de la création, de la construction » (A4523) ; la clausule «Un matin dans le ciel pour un réveil sans Mères Terribles » préfigure à la fois la "Terrible Mère » de «L'Arbre de Gengis Khan» et les «Enfants sombres. Enfants de la Mère Guerrière » de la « Caste des guerriers ».

De surcroit, et c'est là surtout que réside le caractère essentiel de ce texte, "Les sandales éclatantes ${ }^{31}$, rédigé entre septembre et novembre 1949, constitue pour l'écrivain le "premier poème», c'est-à-dire qu'il marque la limite a quo à partir de laquelle le poète s'est révélé à lui-même dans son plein statut de créateur. À la date du 30 mai 1956, Bauchau note encore :

Parfois, comme pour «Les sandales éclatantes », l'inspiration s'emparait de moi physiquement, me forçait à marcher, agitait mon cœur, me remplissait d'une certitude passionnée mais obscure, d'un sentiment de triomphe qui se fondait en humilité. (A45324)

L'inspiration, cette "certitude passionnée mais obscure», a étreint le poète qui se reconnaît désormais comme tel, et il n'est pas étonnant de voir la matière poétique porter la trace de ce ravissement, par l'entremise du motif des «sandales » (" éclatantes », tout comme la «Circonstance»), symbole de vie et d'élévation mystique ${ }^{32}$ dont s'orne Hermès, gardien des hommes en chemin (« guide antique du voyage », dit le poème), inventeur

\footnotetext{
30 Voir FHB A2143 : «Trois étalons l'écume haut / Foulant sa prison de roseaux / La reine attend le roi nouveau / L'archer vierge suivi par les princes bourreaux // Tirant des flèches de bouleau / Il entre en son palais nouveau / Les quatre vents aux naseaux / Sous senes étendards d'herbe et de sang de taureau ». On notera en outre que dans une première version "Les sandales éclatantes» portait le titre de "Vert de Terre» (FHB A2241).

31 Voir FHB A2025 et FHB A2236 à A2242.

32 Jean Chevallier et Alain Gheerbrant, Dictionnaire des symboles. Mythes, rêves, coutumes, gestes, formes, figures, couleurs, nombres, Paris, Robert Laffont/Jupiter, «Bouquins », p. 843.
} 
d'une lyre et archétype du dieu-enfant ${ }^{33}$. Le retentissement des «Sandales éclatantes » à l'orée de la carrière poétique d'Henry Bauchau est capital et le soin peu habituel que ce dernier plaça dans le passage à la mise au net du poème en est un indice manifeste : en plus d'une calligraphie soignée, le texte fut illustré par Alexis Keunen (1921-1990), en faisant un objet d'art à statut d'exception.

Curieusement, Henry Bauchau associe également l'irruption du « souffle ${ }^{34}$ de l'inspiration à la « chambre blanche »: "Alors viendrait "La chambre blanche", c'est là que j'ai chaussé les sandales éclatantes», poursuit-il dans son journal, le 24 août 1964 (GM, p 151; nous soulignons). Comment comprendre cette affirmation a priori paradoxale? Il faut pour cela interroger le statut symbolique que recèle la «chambre blanche ». Le roman $L a$ Déchirure est à ce propos déterminant: la chambre blanche ${ }^{35}$ est le lieu du « jeu originel» :

C'est alors que nous commencions le jeu, qui était simple comme il convient, mais assorti de règles compliquées qu'il fallait respecter strictement. La première était de se mettre sur le ventre, la figure enfoncée dans l'oreiller, ou bien à genoux, l'oreiller entre les cuisses et le visage sur le matelas. On pouvait alors se frotter doucement le ventre sur le matelas ou l'oreiller. Si on éprouvait une sensation, jointe au sentiment d'avoir réellement produit une chose vivante, on criait : J'ai fait un jeune. [...] On ne produisait pas ce qu'on voulait, il y avait un don, une rencontre. Produire un poulain était l'entreprise la plus difficile, mais rarement couronnée de succès. [...] [C'était] une farce que nous faisions au bon Dieu et aux grandes personnes, qui croyaient s'être réservé exclusivement la puissance de créer [...]. (D03, pp. 180-182.)

La façon dont est compris et caractérisé l'éveil sexuel du jeune garçon est remarquable : ce qui se joue à travers ce souvenir « originel», c'est avant tout la mise en œuvre d'un processus de création. Tout l'enjeu

\footnotetext{
33 Voir Carl Gustav Jung et Charles Kerényi, L'Essence de la mythologie, op. cit.

34 À propos de l'écriture de «L'Arbre de Gengis Khan», Bauchau écrit, le 7 janvier 1956: «Je sens là, mieux qu'ailleurs, que ce souffle ne m’appartient pas, que j’ai été seulement le lieu de son passage. » (FHB A4520.)

35 À l'instar de Blanche Reverchon-Jouve, que Bauchau nomme également «Miss Blanche ».
} 
consiste dans le fait d'expérimenter « la puissance de créer » et c'est en cela que réside la satisfaction : "avoir réellement produit une chose vivante ». Par conséquent «Les sandales éclatantes» symbolise ce retour au «jeu originel » et le cheminement, dont Géologie est le premier aboutissement, correspond à la régression, la lente migration vers l'enfance, c'est-à-dire vers cette capacité naturelle à la création que le poète tente de faire remonter de ses profondeurs. Certes, la recherche de ce «don», de cette «rencontre» est difficile et la "production» ne s'avère pas toujours réussie, surtout s'il s'agit de «l'entreprise la plus difficile », qui requiert la puissance de création la plus importante, à savoir celle du «poulain ». Ainsi comprend-on mieux l'angoisse de la perte d'une inspiration si âprement retrouvée qui transparaît derrière l'alexandrin auquel le poète avait songé pour le titre de son recueil :

Les chevaux de la mer n'auront plus [sic] de poulains », vers donné [déclare Henry Bauchau dans «La circonstance éclatante »], jailli directement des profondeurs, je projette dans l'imaginaire d'un passé ma crainte de voir l'inconscient cesser d'être fertile, cesser de me donner des poèmes à écrire au moment où l'espérance reprend courage et où je commence à m'ouvrir à la fécondité du dialogue intérieur. (EE, p. 25.)

S'il n'est pas étonnant d'assister à une réduction de la matière poétique première dans le cadre de la phase rédactionnelle $e^{36}$, il est cependant plus rare que ce travail d'élagage se poursuive autant au cours de la phase éditoriale. Le travail, l'effort poétique d'Henry Bauchau est sans fin et jamais le cisèlement du poème et du recueil ne semble parvenu à son degré de perfection ultime. Autrement dit, le «travail vivant de la poésie », expression que nous reprenons à Jérôme Thélot ${ }^{37}$, est, de fait, un travail vivant, et jamais la matière n'acquiert le statut d'objet. En effet, jamais celle-ci n'est objectée, ne devient un élément posé une fois pour toute, séparé de la chair du poète. Au contraire, elle

\footnotetext{
${ }^{36}$ En général, « une fois le mouvement d'amplification parvenu à son maximum, on note un renversement de tendance et une réduction d'environ $40 \%$ de la masse textuelle » Pierre-Marc De Biasi, Génétique des textes, Paris, C.N.R.S. éditions, "Biblis », 2011, p. 97.

37 Jérôme Thélot, Le Travail vivant de la poésie, Paris, Les Belles Lettres, «Encre marine », 2013.
} 
apparaît comme faisant partie constitutivement de l'écrivain, découvrant une relation d'affectivité au poème sans cesse renégociée. En ce sens, la poésie pour Bauchau n'est pas simple occupation littéraire, mais se comprend "comme l'acte propre de la vie humaine, comme le faire même en lequel cette vie consiste, comme l'édification par excellence de son être de vie $»^{38}$.

Si cette nécessité vitale du dire poétique a en permanence accompagné Henry Bauchau, il nous semble que ses enjeux se sont révélés particulièrement cruciaux dans l'après-guerre, au moment où l'écrivain se cherchait une voie de reconstruction personnelle. Plongeant ses racines dans les tréfonds de son enfance, le poète s'engage à cette époque dans une lutte charnelle avec l'écriture, où obscurité et lumière se mêlent inlassablement avant de finalement le laisser épuisé, à l'aube, dans la «Vérité du poème » :

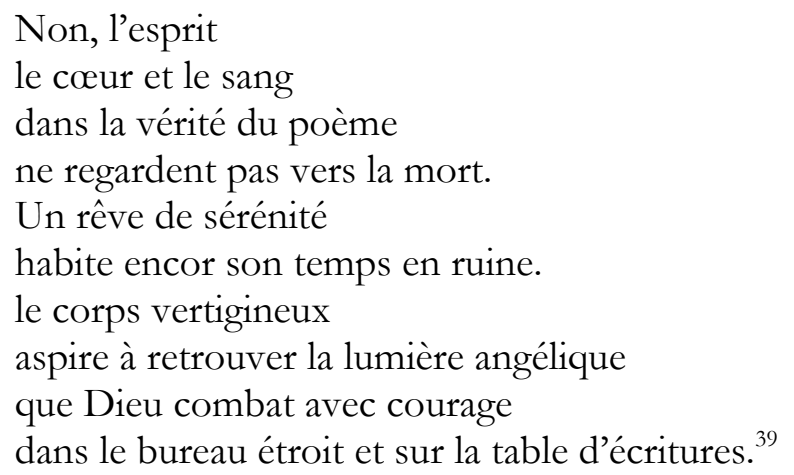

Jérémy LAMBERT

Université catholique de Louvain

\footnotetext{
${ }^{38}$ Ibid., p. 17.

${ }^{39}$ FHB A2112. Le poème est daté de 1989 (il est alors intitulé «La table »). On en trouve les premières ébauches en dates des 24 et 28 décembre 1989, dans le journal de création de l'écrivain ( $J A$, pp. 20-22). Il sera finalement publié, sous le titre «La table d'écriture ", en 2002, dans la revue Écriture (nº0, p. 28).
} 